\title{
Some aspects of lubrication in heavy regimes: Thermal effects, stability and turbulence ${ }^{\text {th }}$
}

\author{
J. Durany *, J. Pereira-Pérez, F. Varas \\ Dep. Matemática Aplicada II, E.I. Telecomunicación, Universidad de Vigo, Campus Marcosende, 36310 Vigo, Spain \\ Received 28 October 2011; received in revised form 28 August 2013; accepted 9 September 2013 \\ Available online 16 September 2013
}

\begin{abstract}
In this work, a combination of numerical methods applied to thermohydrodynamic lubrication problems with cavitation is presented. It should be emphasized the difficulty of the nonlinear mathematical coupled model involving a free boundary problem, but also the simplicity of the algorithms employed to solve it. So, finite element discretizations for the hydrodynamic and thermal equations combined with upwind techniques for the convection terms and duality methods for nonlinear features are proposed.

Additionally, a model describing the movement of the shaft is provided. Considering the shaft as a rigid body this model will consist of an ODE system relating acceleration of the center of gravity and external and pressure loads. The numerical experiments of mechanical stability try to clarify the position of the neutral stability curve.

Finally, a rotating machine for ship propulsion involving both axial and radial bearings operating with nonconventional lubricants (seawater to avoid environmental pollution) is analyzed by using laminar and turbulent inertial flows.

(C) 2013 IMACS. Published by Elsevier B.V. All rights reserved.
\end{abstract}

Keywords: Lubrication; Thermohydrodynamic; Stability; Turbulent flows

\section{Introduction}

The analysis of thermal effects in lubrication problems becomes very important when the device operates under high rotation speed and considerable imposed loads (see [7], among others, and references therein). In those situations, the dissipated energy by viscous effects is significant and causes a temperature raise that results in a decrease of the lubricant viscosity. Consequently, the thermohydrodynamic coupling is driven by the viscosity influence in the hydrodynamic equation, and the velocity field obtained from pressure gradients that is introduced in the energy equation.

On the other hand, in dynamical regimes it exists a wide variety of literature devoted to the stability analysis of journal bearings in different cases and mechanical devices (employing analytical methods $[14,16,19,20]$, or numerical techniques [17,18,21], among others). It is well known, from the analysis of simplified models, that this journal-bearing system can exhibit a limit-cycle response for certain values of the working parameters (see [9]). The contribution here 

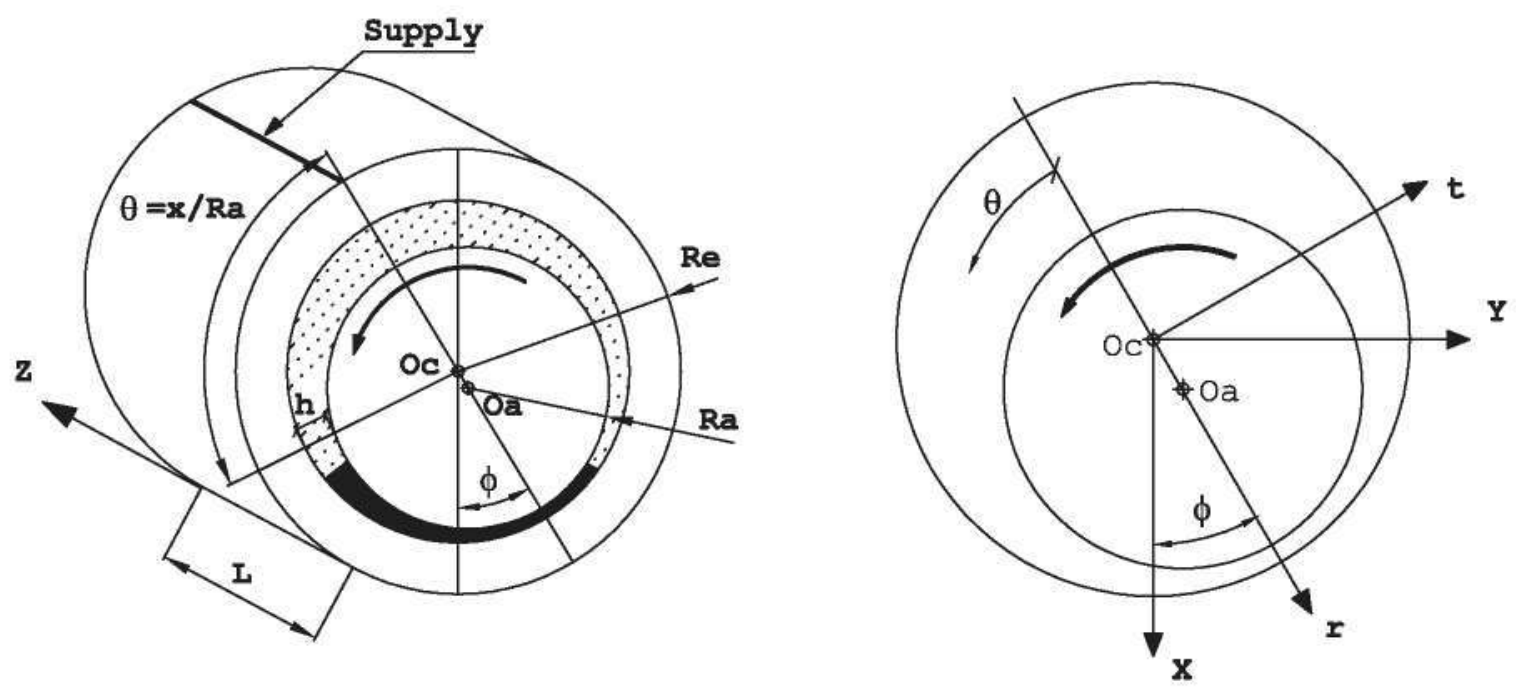

Fig. 1. Journal-bearing.

proposed is the development of a numerical algorithm able to efficiently integrate the complete transient thermohydrodynamic model (including cavitation phenomena) in order to predict the journal-bearing stability properties under more general conditions.

For numerical purposes, the solution of the free boundary problem associated to the Elrod-Adams cavitation model (see $([1,2]$, for details) is obtained by means of a duality method applied to a maximal monotone operator, and a finite element spatial discretization ([3,6], and references therein). The energy equation in the lubricant film is solved with first order finite element schemes due to the simple upwinding used for convective terms. Finally, the resulting semidiscretized in space dynamical model consist of a DAE-like system in which algebraic equations are replaced by an inclusion given by the relation between pressure and lubricant fluid concentration in Elrod-Adams model (see $[9,20])$. As a consequence time-integration should be done with implicit schemes because the system could present an oscillatory behavior, and the coupled problem to be solved at each time step must be segregated (see [9] for details).

The above models and the proposed numerical schemes are used to analyze the operational conditions of industrial plain bearings. More precisely, a real application involving a radial journal bearing and a thrust pad bearing working in rotating machines for ship propulsion has been carefully simulated. In this case, since the devices are submerged under water, then the small viscosity of seawater as lubricating fluid is an additional difficulty. Moreover, due to larger Reynolds numbers, it requires a more critical analysis of minimum lubricant film thickness and load carrying capacity, involving the possible influence of inertial forces and the presence of turbulence in the lubricating film. A model, introduced by Constantinescu-Galetuse [5], which defines the turbulent viscosity at every point of the film based on predominant Couette flow using average velocities, is used to take into account this new feature. Thus, the influence of the laminar or turbulent regime on bearings performance is also provided for this industrial application.

\section{Thermohydrodynamic laminar model in radial bearings}

This section recalls the thermohydrodynamic classical model used in radial bearing lubrication analysis (see [7,11], among others).

A coordinate system in which $\theta$ represents a circumferential coordinate, $y$ a (local) coordinate across the fluid film, and $z$ the axial coordinate is used (as indicated in Fig. 1). The origin of coordinate $\theta$ is assumed over the centers line, $\overline{O_{c} O_{a}}$, so that the minimum gap of the device is placed at the angle $\theta=\pi$. On the other hand, the reference $y=0$ is taken on the bush surface. 


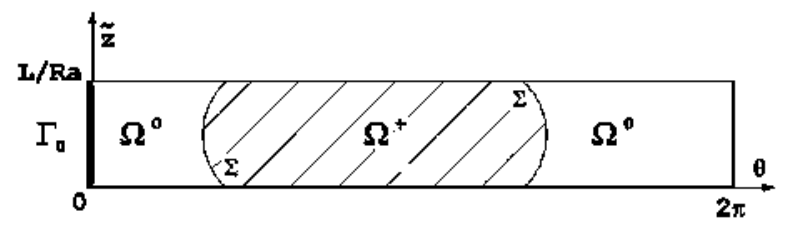

Fig. 2. Hydrodynamic domain configuration.

The non-dimensional fluid domain, corresponding to the mean surface between the shaft and the bushing, is transformed into $\Omega=[0,2 \pi] \times\left[0, L R_{a}\right]$, where $R_{a}$ and $L$ denote the radius and the shaft length, for the $(\theta, \tilde{z})$ coordinates, and the height:

$$
\bar{h}=1+\varepsilon \cos \theta,
$$

where $\varepsilon$, the journal-bearing eccentricity coefficient, must be computed by solving a mechanical problem related to the load acting on the shaft.

Due to the small fluid film thickness, the classical hydrodynamic model is given by Reynolds equation for Newtonian fluids with constant density (see [11], among others). Moreover, the mathematical formulation of the cavitation moving boundary problem is posed by means of the so called Elrod-Adams model [10]. It introduces the lubricating fluid concentration, $\vartheta$, as an additional unknown to the original problem, that takes the value 1 for the fluid part, $\Omega^{+}$, and takes any value between 0 and 1 for the cavitated one, $\Omega^{0}$ (see Fig. 2). Thus, the hydrodynamic problem can be written as follows.

Find the pressure and concentration, $(\bar{p}, y)$, such that:

$$
\begin{aligned}
& \frac{\partial}{\partial \theta}\left(\bar{h}^{3} \bar{G} \frac{\partial \bar{p}}{\partial \theta}\right)+\frac{\partial}{\partial \tilde{z}}\left(\bar{h}^{3} \bar{G} \frac{\partial \bar{p}}{\partial \tilde{z}}\right)=\frac{\partial}{\partial \theta}\left(\bar{h}\left(1-\frac{\bar{I}_{2}}{\bar{J}_{2}}\right)\right) \\
& +\frac{1}{\omega} \frac{\partial(\vartheta \bar{h})}{\partial t}, \quad \bar{p}>0 \text { and } \vartheta=1 \quad \text { in } \Omega^{+} \times\left[0, t_{F}\right], \\
& \frac{\partial}{\partial \theta}\left(\vartheta \bar{h}\left(1-\frac{\bar{I}_{2}}{\bar{J}_{2}}\right)\right)+\frac{1}{\omega} \frac{\partial \vartheta \bar{h})}{\partial t}=0, \quad \bar{p}=0, \quad 0 \leq \vartheta \leq 1 \quad \text { in } \quad \Omega^{0} \times\left[0, t_{F}\right], \\
& \bar{h}^{3} \bar{G} \frac{\partial \bar{p}}{\partial \vec{n}}=(1-\vartheta) \bar{h}\left(1-\frac{\bar{I}_{2}}{\bar{J}_{2}}\right) \cos (\vec{n}, \vec{i}), \quad \bar{p}=0 \quad \text { on } \Sigma \times\left[0, t_{F}\right], \\
& \bar{p}=0 \text { on } \partial \Omega \times\left[0, t_{F}\right], \vartheta=\vartheta_{0} \text { on } \Gamma_{0} \times\left[0, t_{F}\right], \\
& \vartheta(0, \theta, \overline{\mathrm{z}})=\vartheta^{0}(\theta, \tilde{z}),
\end{aligned}
$$

where $\omega$ is the rotation speed. Moreover, $\Sigma$ represents the free boundary between the lubricated region $\left(\Omega^{+}\right)$and the cavitated one $\left(\Omega^{0}\right), \vec{n}$ a normal vector to $\Sigma, \vec{i}$ the unitary normal vector pointing to $\theta$-direction, $\Gamma_{0}$ the boundary where fluid is supplied through (located at $\theta=0$ ), and $t_{F}$ the final time. The mathematical model (2)-(6) has been widely and thoroughly analyzed by Bayada et al. Several results of existence and uniqueness of solution to stationary and evolutionary problems, for different boundary conditions, the asymptotic derivation of this model and some of its variants, along with many other properties related to the referred model, can be seen in [1-4] (just to mention some references) and references therein (precisely, the authors thank professor Bayada for his great scientific advice throughout these years).

The viscosity coefficients $\bar{G}, \bar{I}_{2}$ and $\bar{J}_{2}$ are given by:

$$
\bar{G}=\int_{0}^{1} \frac{\bar{v}}{\bar{\mu}}\left(\bar{y}-\frac{\bar{I}_{2}}{\bar{J}_{2}}\right) d \bar{y}, \quad \bar{I}_{2}=\int_{0}^{1} \frac{\bar{v}}{\bar{\mu}} d \bar{y}, \quad \bar{J}_{2}=\int_{0}^{1} \frac{d \bar{y}}{\bar{\mu}},
$$

where the fluid viscosity, $\bar{\mu}$, depends on the fluid temperature, $T_{j}$, following the behavior law:

$$
\bar{\mu}=e^{-\beta T_{0}\left(\bar{T}_{f}-1\right)}
$$


with $\beta$ denoting a thermoviscous coefficient and $T_{0}$ a reference temperature. The dimensionless velocity components of the lubricant are given by:

$$
\bar{u}=-\bar{h}^{2} \frac{\partial \bar{p}}{\partial \phi}\left(\frac{\bar{I}_{2}}{\bar{J}_{2}} \bar{J}-\bar{I}\right)+\frac{\bar{J}}{\bar{J}_{2}}, \quad \bar{w}=-\bar{h}^{2} \frac{\partial \bar{p}}{\partial \bar{z}}\left(\frac{\bar{I}_{2}}{\bar{J}_{2}} \bar{J}-\bar{I}\right),
$$

where $\bar{I}, \bar{J}$, are defined by:

$$
\bar{l}=\int_{0}^{\bar{y}} \frac{\xi}{\bar{\mu}} d \xi . \quad \bar{J}=\int_{0}^{\bar{y}} \frac{d \xi}{\bar{\mu}} .
$$

For the thermal problem, it is considered that the reached temperatures are not large enough to take into account heat flows from the fluid film to the shaft and bush. This simplification avoids solving a coupled thermal problem in these two devices and limits the study to the dimensionless energy equation in the fluid (see [7]):

$$
\rho c_{p} \frac{C^{2}}{k} \frac{\partial \overline{T_{f}}}{\partial t}+P_{e}\left(\bar{u} \frac{\partial \overline{T_{f}}}{\partial \theta}+\bar{w} \frac{\partial \overline{T_{f}}}{\partial \bar{z}}\right)=\frac{1}{\bar{h}^{2}}\left(\frac{\partial^{2} \overline{T_{f}}}{\partial \theta^{2}}+\frac{\partial^{2} \overline{T_{f}}}{\partial \bar{z}^{2}}\right)+N_{d} \frac{\bar{\mu}}{\bar{h}^{2}}\left[\left(\frac{\partial \bar{u}}{\partial \bar{y}}\right)^{2}+\left(\frac{\partial \bar{w}}{\partial \bar{y}}\right)^{2}\right],
$$

where $\overline{T_{f}}$ is the non-dimensional temperature, $T_{f}=T_{0} \quad \overline{T_{f}}$, with reference temperature $T_{0}$. Moreover, $P_{e}$ and $N_{d}$ are the Peclet and Brinkman numbers, respectively, and given by:

$$
P_{e}=\frac{\omega C^{2}\left[\rho_{f} c_{f} \vartheta+\rho_{a} c_{a}(1-\vartheta)\right]}{\left[k_{f} \vartheta+k_{a}(1-\vartheta)\right]}, \quad N_{d}=\frac{\mu_{0} \vartheta \omega^{2} R_{a}^{2}}{\left[k_{f} \vartheta+k_{a}(1-\vartheta)\right] T_{0}}
$$

being the coefficients:

$$
\rho c_{p}=\rho_{f} c_{f} \vartheta+\rho_{a} c_{a}(1-\vartheta), k=k_{f} \vartheta+k_{a}(1-\vartheta),
$$

with $\rho_{f}, c_{f}, k_{f}, \rho_{a}, c_{a}, k_{a}$ denoting the averaged density, specific heat and thermal conductivity of the fluid and gas, respectively (see [11], Chapter 10 ), $C$ the clearance of the journal-bearing and $\mu_{0}$ the viscosity at the reference temperature $T_{0}$.

Eq. (11) must be completed with Dirichlet boundary conditions on the supply groove (known temperature) or with Neumann boundary conditions (known heat flow). Additionally, an initial condition must be added in this timedependent problem.

The dynamical model for the shaft motion is based on Newton's second law, that governs the displacement of the gravity center of the shaft. This law is modified by introducing non-dimensional values. Assuming a fixed shaft in the $z$-coordinate, and scaling forces with the modulus $\left(W_{0}\right)$ of external load, the dynamical equation can be written as follows (see [11], among others):

$$
\frac{M}{W_{0}}\left[\begin{array}{l}
a_{X} \\
a_{Y}
\end{array}\right]=\left[\begin{array}{l}
\bar{W}_{X} \\
\bar{W}_{Y}
\end{array}\right]+\left[\begin{array}{l}
\bar{F}_{X} \\
\bar{F}_{Y}
\end{array}\right] .
$$

where $M$ denotes the mass of the shaft, $\vec{a}=\left(a_{X}, a_{Y}\right)$ the acceleration of its center, $\vec{W}_{0}=\left(\bar{W}_{X}, \bar{W}_{Y}\right)$ the applied external load, $\vec{F}=\left(\bar{F}_{X}, \bar{F}_{Y}\right)$ the fluid reaction force, and subindexes ' $X$ ' and ' $Y$ ' show the corresponding components of the vectors. The force $\vec{F}$ is given by the integration of the force density, computed from the stress tensor in the fluid (this tensor being composed by a pressure component and a viscous one), on the fluid-shaft boundary (see [11], Chapter 6 , for details). Moreover, it must be kept in mind that this fluid reaction force $\vec{F}$ in $\mathrm{Eq}$. (14) depends (through the solution of the hydrodynamic problem associated to the lubricant film) on both the position and the velocity of the shaft (observe that the height of the fluid film, $\bar{h}$, depends on the shaft position).

On the other hand, the shatt center position $(X, Y)$, and time $t$ are scaled following the relations:

$$
X=C \bar{X}, \quad Y=C \bar{Y}, \quad t=\frac{\tau}{\omega},
$$

where $(\bar{X}, \bar{Y})$ and $\tau$ are the dimensionless position and time, respectively. 

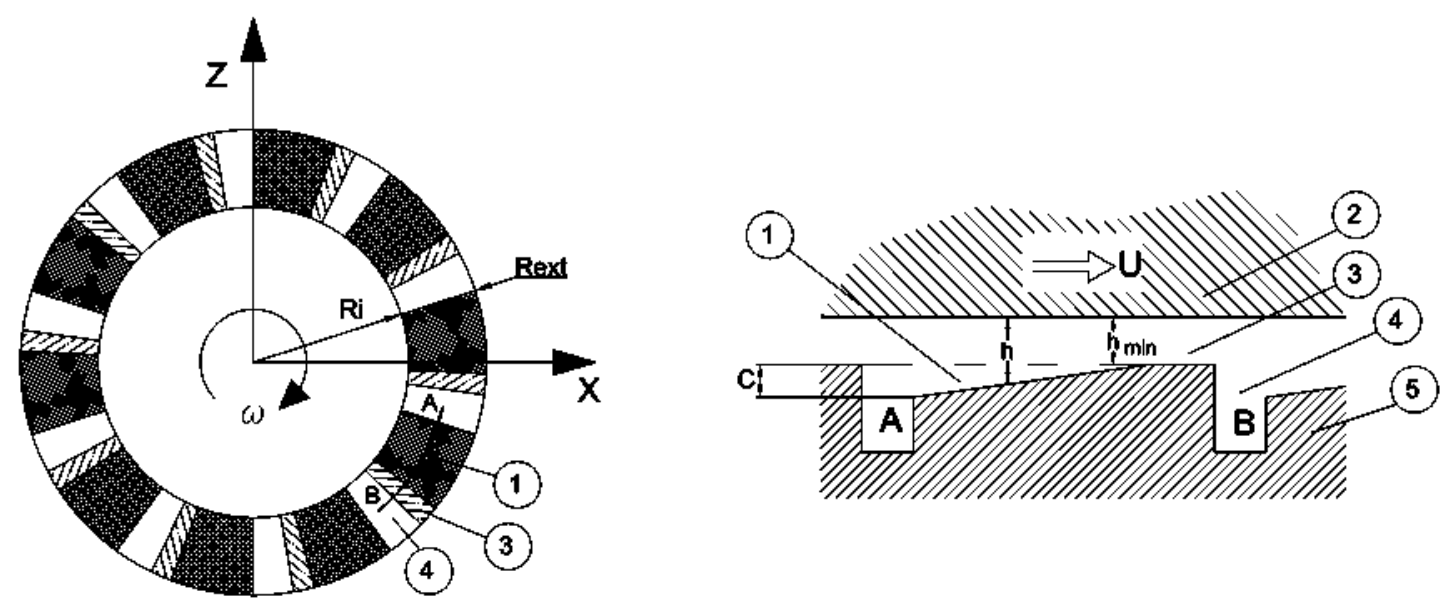

Fig. 3. Thrust pad bearing (pad geometry ISO-12131): (1) wedge surface, (2) thrust collat, (3) supporting surface, (4) lubrication groove, and (5) thrust bearing ring.

Substituting the new variables in (14), the resulting nondimensional equation is obtained:

$$
\bar{M}\left[\begin{array}{c}
\ddot{\bar{X}} \\
\ddot{\bar{Y}}
\end{array}\right]=\left[\begin{array}{c}
\bar{W}_{\bar{X}} \\
\bar{W}_{\bar{Y}}
\end{array}\right]+\left[\begin{array}{c}
\bar{F}_{\bar{X}} \\
\bar{F}_{\bar{Y}}
\end{array}\right],
$$

where factor $\bar{M}=\left(\left(M C \omega^{2}\right) / W_{0}\right)$ is the shaft dimensionless mass.

\section{Thermohydrodynamic laminar model in pad bearings}

In this section, the classical equations related to laminar hydrodynamic models in pad bearings (see Fig. 3, where a highly rotating thrust collar is supported by the pads of the bearing) are remembered. The Reynolds equation with the cavitation model similar to (2)-6) in the plane $(x, z)$ is given by (see [11], among others):

$$
\frac{\partial}{\partial x}\left(\rho G \frac{\partial p}{\partial x}\right)+\frac{\partial}{\partial z}\left(\rho G \frac{\partial p}{\partial z}\right)=\frac{\partial}{\partial x}\left(\rho u_{2}\left(h-\frac{I_{2}}{J_{2}}\right)\right)+\frac{\partial}{\partial z}\left(\rho u_{2}\left(h-\frac{I_{2}}{J_{2}}\right)\right)+\rho \frac{\partial(h \vartheta)}{\partial t}
$$

$p$ being the fluid pressure, $\rho$ the constant density, $G$ the flux viscosity coefficient, $\left(u_{2}, w_{2}\right)$ the components of the bearing rotational speed, $h$ the film fluid thickness and $J_{2}, I_{2}$, two viscosity coefficients to be defined later in (20) and (21), respectively.

For computational simplicity, a dimensionless problem in one pad (from zone A to B in Fig. 3) is posed. For this purpose, the following dimensionless variables and parameters are introduced:

$$
\begin{aligned}
& x=R_{i} \bar{x}, \quad z=R_{i} \bar{z}, \quad y=h \bar{y}, \quad h=C \bar{h}, \\
& p=\mu_{0} \omega\left(\frac{R_{i}}{C}\right)^{2} \bar{p}, \quad \mu=\mu_{0} \bar{\mu}, \quad u=\omega R_{i} \bar{u}, \quad w=\omega R_{i} \bar{w},
\end{aligned}
$$

where $R_{i}$ represents the pad internal radius, $C$ is the gap and $h$ the thickness, according the geometry ISO-12131 (see schematic view of a thrust pad bearing in Fig. 3). Moreover, $\mu_{0}$ is the reference fluid viscosity on the supply groove, $(u, w)$ are the velocity components and $\omega$ is the rotational speed of the axial bearing. Taking this into account and that viscosity is constant respect to coordinate $y$, the coefficients $J_{2}$ and $I_{2}$ are respectively defined by:

$$
\begin{aligned}
& J_{2}=\int_{0}^{h} \frac{1}{\mu} d y=\int_{0}^{1} \frac{1}{\mu_{0} \bar{\mu}} h d \bar{v}=\frac{h}{\mu_{0}} \quad \frac{1}{\bar{\mu}}=\frac{c \bar{h}}{\mu_{0}} \frac{1}{\bar{\mu}}, \\
& I_{2}=\int_{0}^{h} \frac{y}{\mu} d y=\int_{0}^{1} \frac{h \bar{y}}{\mu_{0} \bar{\mu}} h d \bar{y}=\frac{h^{2}}{\mu_{0}} \frac{1}{2 \bar{\mu}}=\frac{C^{2} \bar{h}^{2}}{\mu_{0}} \frac{1}{2 \bar{\mu}} .
\end{aligned}
$$


Then, the flow coefficient can be written as:

$$
G=\int_{0}^{h} y\left(y-\frac{I_{2}}{J_{2}}\right) \frac{1}{\mu} d y=\int_{0}^{1} C \bar{h} \bar{y}\left(C \bar{h} \bar{y}-C \bar{h} \frac{1}{2}\right) \frac{1}{\mu_{0} \bar{\mu}} C \bar{h} d \bar{y},
$$

that is:

$$
G=\frac{C^{3} \bar{h}^{3}}{\mu_{0} \bar{\mu}} \int_{0}^{1} \bar{y}\left(\bar{y}-\frac{1}{2}\right) d \bar{y}=\frac{C^{3} \bar{h}^{3}}{\mu_{0} \bar{\mu}} \frac{1}{12} .
$$

By applying changes of variable (18) and (19) and expressions (20), (21) and (23) to (17), the following nondimensional Reynolds equation is obtained:

$$
\frac{\partial}{\partial \bar{x}}\left(\frac{\bar{h}^{3}}{12 \bar{\mu}} \frac{\partial \bar{p}}{\partial \bar{x}}\right)+\frac{\partial}{\partial \bar{z}}\left(\frac{\bar{h}^{3}}{12 \bar{\mu}} \frac{\partial \bar{p}}{\partial \bar{z}}\right)=\frac{\partial}{\partial \bar{x}}\left(\bar{u}_{2} \frac{\bar{h}}{2}\right)+\frac{\partial}{\partial \bar{z}}\left(\bar{w}_{2} \frac{\bar{h}}{2}\right)+\frac{1}{\omega} \frac{\partial(\bar{h} \vartheta)}{\partial t},
$$

and the dimensionless velocity components are given by:

$$
\bar{u}=-\bar{h}^{2} \frac{\partial \bar{p}}{\partial \bar{x}}\left(\frac{\bar{I}_{2}}{\bar{J}_{2}} \bar{J}-\bar{I}\right)+\bar{u}_{2} \frac{\bar{J}}{\bar{J}_{2}}, \quad \bar{w}=-\bar{h}^{2} \frac{\partial \bar{p}}{\partial \bar{z}}\left(\frac{\bar{I}_{2}}{\bar{J}_{2}} \bar{J}-\bar{I}\right)+\bar{w}_{2} \frac{\bar{J}}{\bar{J}_{2}},
$$

where $\bar{J}, \bar{I}$ denote integrals defined as $\overline{\boldsymbol{J}}_{2}, \overline{\boldsymbol{I}}_{2}$ (see (20) and (21)), respectively, where now the integration limits are 0 and $\bar{y}$. Eqs. (24) and (25) are completed with boundary and initial conditions.

For the fluid thermal problem, the non-dimensional energy Eq. (11) is posed in coordinates $(\bar{x}, \bar{z})$. The coefficients $P_{e}, N_{d}$ in Eq. (11) are given in (12), but changing the pad internal radius $R_{i}$ by $R_{a}$. Eq. (11) is also completed with Dirichlet boundary condition on the supply groove (known temperature) and with the initial condition.

\section{Numerical methods and stability curve}

The numerical solution of (16), coupled with the thermohydrodynamic fluid problem, could present an oscillatory behavior. That is the reason for choosing a backward Euler method. At each time step, this method solves the nonlinear system resulting from time discretization of Eq. (16) by using the shaft center position, $d^{n+1}=(\bar{X}, \bar{Y})$, and velocity, $v^{n+1}=(\dot{\bar{X}}, \dot{\bar{Y}})$, as unknowns:

$$
\frac{d^{n+1}-d^{n}}{\Delta t}-v^{n+1}=0, \quad \bar{M}\left[\frac{v^{n+1}-v^{n}}{\Delta t}\right]-\bar{W}_{0}-\bar{F}=0,
$$

jointly with the solution $(\bar{p}, \vartheta)$ of the Elrod-Adams cavitation problem (2)-(6), and the corresponding equation for temperature (11).

In order to solve this nonlinear system with variables $\left(d, v, \bar{p}, \vartheta, \overline{T_{f}}\right)$, a quasi-Newton method that approximates the Jacobian matrix is chosen. More precisely, Broyden method combined with the Armijo-Goldstein criterion to choose the descent direction parameter is used (see [9] for details).

The problem (2)-(6) is solved by using an algorithm based on the following elements (see [8] for details): first, a suitable variational formulation in terms of the multivalued Heaviside operator $H$ is posed to take into account the cavitation feature; next, rewriting temporal and convective terms of (2) in terms of the total derivative is used; then, an upwind scheme of characteristics can be applied to discretize this total derivative following the velocity field; finally, the nonlinear behavior of the multivalued Heaviside operator, $H$, can be solved at each iteration of the time-algorithm by using a duality type method when it is applied to a monotone maximal operator. It consists of another iterative procedure to obtain the numerical solution of the free boundary problem by introducing Lagrange multipliers, and consequently it is possible to linearize the Heaviside function by using Yosida approximations. On the other hand, Lagrange $P_{1}$ finite elements for the spatial approximation are introduced.

Moreover, in order to build the corresponding blocks of the fluid thermal problem in the system matrix, a finite element discretization of Eq. (11) is applied. In this way, the nonlinear behavior of the dissipation term involving the viscosity, $\bar{\mu}$, is treated explicitly at each time step (see [7] for details).

Searching conditions such that the system (26) has a family of periodic solutions from perturbation of the stationary solution, the Routh-Hurwitz criterion provides an existence condition. This condition states the existence of a critical 


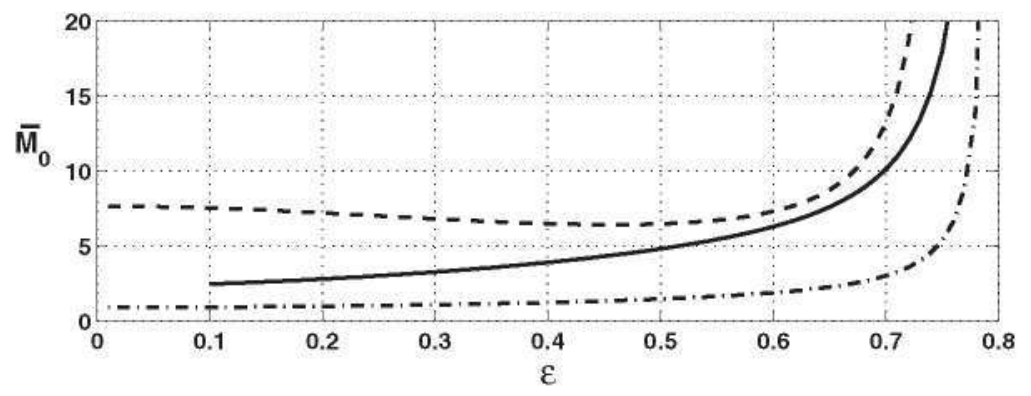

Fig. 4. Stability curves. Critical mass $\bar{M}_{0}$ versus $\varepsilon$. Elrod-Adams model (fixed axis system and $\bar{p}_{0}=0$ on $\theta=0$, solid line), classical analytical short bearing model (dashed line), and classical analytical long bearing model (dash-dotted line).

velocity, $\tilde{\omega}_{0}$, (or equivalently, critical mass $\bar{M}_{0}$ ), for the dimensionless rotation speed of the shaft (and consequently for the dimensionless mass), given by (see [9]):

$$
\tilde{\omega}=\omega \sqrt{\frac{M C}{W_{0}}}=\omega \sqrt{\bar{M}} .
$$

It is proved that if $\bar{M}<\bar{M}_{0}$ then the system is stable (when the equilibrium position is perturbed, the system returns to the original position) whereas if $\bar{M}>\bar{M}_{0}$ it becomes unstable (the system being perturbed, the shaft will no longer return to the equilibrium position). This stability change is related to a Hopf bifurcation at $\bar{M}=\bar{M}_{0}$ (see, for instance, [12]) meaning that a branch of periodic solutions will coexist with the stationary solution, either for $\bar{M}<\bar{M}_{0}$ (subcritical Hopf bifurcation) or for $\bar{M}>\bar{M}_{0}$ (supercritical Hopf bifurcation). The behavior of the shaft in the unstable regime $\left(\bar{M}>\bar{M}_{0}\right)$ will be quite different depending on the kind of bifurcation. In the supercritical case, the shaft will evolve toward a limit cycle with small amplitudes if $\bar{M}$ is close to the critical value $\bar{M}_{0}$, whereas, in the subcritical bifurcation, the shaft will leave the vicinity of the steady solution no matter how close mass, $\bar{M}$, is to the critical value. Taking into account the previous explanations and a realistic boundary condition for the hydrodynamic equation (imposing the supply pressure condition, $\bar{p}_{0}=0$, on the fixed supply groove $\theta=0$ ), the solution of the global coupled model in $\left(d, v, \bar{p}, \vartheta, \overline{T_{f}}\right)$ provides the stability curve shown in Fig. 4. In the computation of the neutral stability curve, temporal integration of the problem has been used. Although more expensive (from the computational point of view) than the computation of the eigenvalues of the linearized problem, this integration allows both to reveal the subcritical nature of the Hopf bifurcation and to compute the associated limit cycles. Moreover, the stability margin here obtained is greater than the corresponding one obtained by using the classical long bearing model, but smaller than the margin deduced from the classical short bearing model.

\section{Inertial effects and turbulence}

In this section, a hydrodynamic model following Kosasih-Tieu [13] is proposed. It includes possible effects of fluid inertia and turbulent flow development on axial and radial bearings. This model, in cylindrical coordinates, adapts the Constantinescu-Galetuse [5] turbulence model for axial bearings, including inertial effects. For the sake of simplicity we assume here that no cavitation occurs in the fluid. This assumption will be fully justified for the industrial problem considered below (see Section 6) were modeling of inertia and turbulence effects is needed. So, the equation, in stationary form for simplicity, is:

$$
\frac{\partial}{\partial \theta}\left(\frac{h^{3} G_{\theta}}{r} \frac{\partial p}{\partial \theta}\right)+\frac{\partial}{\partial r}\left(r h^{3} G_{r} \frac{\partial p}{\partial r}\right)=\frac{\partial}{\partial \theta}\left(\frac{U h}{2}\right)-\frac{\partial}{\partial \theta}\left(h^{2} G_{\theta} \rho I_{\theta}\right)-\frac{\partial}{\partial r}\left(r h^{2} G_{r} \rho I_{r}\right),
$$

where $G_{\theta}, G_{r}$, denote the viscosity coefficients in cylindrical coordinates, $U$ is the thrust collar velocity, and $I_{\theta}, I_{r}$, are combination of inertial momentum derivatives, as detailed later.

The following scalings are applied to obtain (28) in non-dimensional form:

$$
U=\omega r \bar{u}, \quad U_{\theta}=\omega \quad R_{i} \bar{u}_{\theta}, \quad U_{r}=\omega R_{i} \bar{u}_{r}, \quad h=C \bar{h}, \quad r=R_{i} \bar{r},
$$




$$
p=\mu_{0} \omega\left(\frac{R_{i}}{C}\right)^{2} \bar{p}, \quad I_{\theta}=\bar{I}_{\theta} \omega^{2} R_{i} C, \quad I_{r}=\bar{I}_{r} \omega^{2} R_{i} C,
$$

being $U_{\theta}, U_{r}$ the averaged fluid velocities. Thus, the terms in (28) can be written as follows:

$$
\begin{aligned}
& \frac{\partial}{\partial \theta}\left(\frac{h^{3}}{r} G_{\theta} \frac{\partial p}{\partial \theta}\right)=\omega R_{i} C \frac{\partial}{\partial \theta}\left(\frac{\bar{h}^{3}}{\bar{r}} \bar{G}_{\theta} \frac{\partial \bar{p}}{\partial \theta}\right), \\
& \frac{\partial}{\partial r}\left(\begin{array}{lll}
r & h^{3} & G_{r} \\
\partial r
\end{array}\right)=\omega R_{i} C \frac{\partial}{\partial \bar{r}}\left(\bar{r} \bar{h}^{3} \bar{G}_{r} \frac{\partial \bar{p}}{\partial \bar{r}}\right) . \\
& \frac{U}{2} \frac{\partial h}{\partial \theta}=\omega R_{i} C \frac{\bar{u}}{2} \frac{\partial \bar{h}}{\partial \theta}, \\
& \frac{\partial}{\partial \theta}\left(h^{2} G_{\theta} \rho I_{\theta}\right)=\omega R_{i} C \overbrace{\left[\frac{\rho \omega R_{i} C}{\mu_{0}}\right]}^{R e^{*}}\left(\frac{C}{R_{i}}\right) \frac{\partial}{\partial \theta}\left(\bar{h}^{2} \bar{G}_{\theta} \bar{I}_{\theta}\right), \\
& \frac{\partial}{\partial r}\left(r h^{2} G_{r} \rho I_{r}\right)=\omega R_{i} C \overbrace{\left[\frac{\rho \omega R_{i} C}{\mu_{0}}\right]}^{R e^{*}}\left(\frac{C}{R_{i}}\right) \frac{\partial}{\partial \bar{r}}\left(\bar{r}^{2} \bar{G}^{2} \bar{G}_{r}\right),
\end{aligned}
$$

and, after dividing by $\omega R_{i} C$, the non-dimensional Reynolds equation is written as:

$$
\frac{\partial}{\partial \theta}\left(\frac{\bar{h}^{3}}{\bar{r}} \bar{G}_{\theta} \frac{\partial \bar{p}}{\partial \theta}\right)+\frac{\partial}{\partial \bar{r}}\left(\bar{r} \bar{h}^{3} \bar{G}_{r} \frac{\partial \bar{p}}{\partial \bar{r}}\right)=\frac{\bar{r}}{2} \frac{\partial \bar{h}}{\partial \theta}-R^{*}\left(\frac{C}{R_{i}}\right) \frac{\partial}{\partial \theta}\left(\bar{h}^{2} \bar{G}_{\theta} \bar{I}_{\theta}\right)-\operatorname{Re}\left(\frac{C}{R_{i}}\right) \frac{\partial}{\partial \bar{r}}\left(\bar{r} \bar{h}^{2} \bar{G}_{r} \bar{I}_{r}\right)
$$

where $R e^{*}=\left(\left(\rho \omega R_{i} C\right) / \mu_{0}\right)$ is the Reynolds number using $C$ (the Reynolds number using $h$ is $R e=\left(\left(\rho \omega R_{i} h\right) / \mu_{0}\right)$, being in this case $h$ an unknown of the problem and position dependent). Coefficients $\bar{G}_{\theta}$ and $\bar{G}_{r}$ correspond to turbulent or laminar flow, depending on the Reynolds number Re. The expressions of these coefficients for the turbulent case can be found later in this section (for the laminar case they are the same ones used in previous sections).

In order to solve the hydrodynamic Eq. (36), the new terms $\left(\bar{I}_{\theta}, \bar{I}_{r}\right)$ on the right hand side of Reynolds equation have to be analyzed. To determine these moments, a known velocity profile is required, and thus a wall law. Constantinescu-Galetuse [5] and Kosasih-Tieu [13] (and other papers in the same line) propose relations between moments and averaged velocities where some coefficients $(\alpha, \beta, \gamma, \ldots)$ are obtained from experimental data. That is:

$$
\begin{aligned}
& I_{\theta \theta}=\int_{0}^{h} u_{\theta} u_{\theta} d z=\alpha U_{\theta}^{2} h+\beta U^{2} h-\gamma U_{\theta} U h, \\
& I_{\theta r}=\int_{0}^{h} u_{\theta} u_{r} d z=\alpha^{\prime} U_{\theta} U_{r} h-\gamma^{\prime} U_{r} U h, \\
& I_{r r}=\int_{0}^{h} u_{r} u_{r} d z=\alpha^{\prime \prime} U_{r}^{2} h,
\end{aligned}
$$

where it is assumed that moments are proportional to the product of averaged velocities along the considered directions $\left(I_{i j} \sim U_{i} U_{j}\right)$. Taking the previous relations as a valid hypothesis, a non-dimensional system can be posed with pressure, averaged velocities and moments as unknowns:

$$
\frac{\partial}{\partial \theta}\left(\frac{\bar{h}^{3}}{\bar{r}} \bar{G}_{\theta} \frac{\partial \bar{p}}{\partial \theta}\right)+\frac{\partial}{\partial \bar{r}}\left(\bar{r} \bar{h}^{3} \bar{G}_{r} \frac{\partial \bar{p}}{\partial \bar{r}}\right)=\frac{\bar{r}}{2} \frac{\partial \bar{h}}{\partial \theta}-R_{e}^{*}\left(\frac{C}{R_{i}}\right) \frac{\partial}{\partial \theta}\left(\bar{h}^{2} \bar{G}_{r_{\theta}} \bar{I}_{\theta}\right)-R_{e}^{*}\left(\frac{C}{R_{i}}\right) \frac{\partial}{\partial \bar{r}}\left(\bar{r}^{2} \bar{G}_{r} \bar{I}_{r}\right),
$$




$$
\begin{aligned}
& \bar{u}_{\theta}=\frac{\bar{r}}{2}-\bar{h}^{2} \bar{G}_{\theta} \frac{1}{\bar{r}} \frac{\partial \bar{p}}{\partial \theta}-R_{e}^{*}\left(\frac{C}{R_{i}}\right) \bar{h} \bar{G}_{\theta} \bar{I}_{\theta}, \\
& \bar{u}_{r}=-\bar{h}^{2} \bar{G}_{r} \frac{\partial \bar{p}}{\partial \bar{r}}-R_{e}^{*}\left(\frac{C}{R_{i}}\right) \bar{h}_{\bar{G}} \bar{G}_{r}, \\
& \bar{I}_{\theta \theta}=\alpha \bar{h} \bar{u}_{\theta}^{2}+\beta \bar{h}^{2}-\gamma \bar{u}_{\theta} \bar{r} \bar{h}, \\
& \bar{I}_{\theta r}=\alpha^{\prime} \bar{u}_{\theta} \bar{u}_{r} \bar{h}-\gamma^{\prime} \bar{u}_{r} \bar{r} \bar{h}, \\
& \bar{I}_{r r}=\alpha^{\prime \prime} \bar{h} \bar{u}_{r}^{2}, \\
& \bar{I}_{\theta}=\left(\frac{1}{\bar{r}} \frac{\partial \bar{I}_{\theta \theta}}{\partial \theta}+\frac{\partial \bar{I}_{\theta r}}{\partial \bar{r}}+\frac{\bar{I}_{\theta r}}{\bar{r}}\right), \quad \bar{I}_{r}=\left(\frac{1}{\bar{r}} \frac{\partial \bar{I}_{\theta r}}{\partial \theta}+\frac{\partial \bar{I}_{r r}}{\partial \bar{r}}-\frac{\bar{I}_{\theta \theta}}{\bar{r}}\right) .
\end{aligned}
$$

On the other hand, considering that these additional terms in (40) should be small corrections on pressure in the Reynolds equation, an iterative procedure to solve the system is performed:

1. Start $\bar{I}_{\theta}$ and $\bar{I}_{r}$ equal to zero.

2. Solve Reynolds equation (40) to obtain $\bar{p}^{i}$ at $i$-iteration.

3. Calculate velocities $\left(\bar{u}_{\theta}^{i}, \bar{u}_{r}^{i}\right)$ in (41) and (42).

4. Calculate moments $\bar{I}_{\theta \theta}^{i}, \bar{I}_{\theta r}^{i}, \bar{I}_{r r}^{i}, \bar{I}_{\theta}^{i}$ and $\bar{I}_{r}^{i}$, applying formulas (43)-(46).

5. If $\left|\bar{p}^{i}-\bar{p}^{i-1}\right| / \bar{p}^{i-1}$ satisfies tolerance test then end the process, otherwise return to step 2 .

In the case of laminar flows, coefficients $\bar{G}_{\theta}$ and $\bar{G}_{r}$ in (40) have analytical expressions related with viscosity and velocity profile on the section. In turbulent flows the viscosity effects are not homogeneous across the section and the velocity profile becomes difficult to compute. So, different turbulent models can be seen in literature. Some of them establish wall laws and compute the velocity profile, others apply experimental formulas. The Boussinesq model is an example of the first group, developing a velocity profile from viscosity variations on the section. A simpler model is used in [5,13], where coefficients $\bar{G}_{\theta}$ and $\bar{G}_{r}$ are related to the Reynolds number on the section. This model considers that it exists a laminar film adjacent to the walls and a full turbulent zone on the middle, but not a transition zone from laminar to turbulent flow. In the laminar zone viscosity is constant, whereas in the turbulent zone it varies with the square root of the distance to the wall and with the velocity gradient. The coefficients of the Reynolds equation thus obtained have been modified to match experimental results. So, for turbulent flows with $10^{3}<\operatorname{Re}<10^{5}$, the works $[5,13]$ propose the following formulas:

$$
\bar{G}_{\theta}=\frac{1}{\left(12+0.0136 R e^{0.9}\right) \bar{\mu}}, \quad \bar{G}_{r}=\frac{1}{\left(12+0.0043 R e^{0.96}\right) \bar{\mu}},
$$

to be applied in Eqs. (40)-(42), because Reynolds numbers for seawater lubricated thrust bearing are in that range $\left(R e=2.2 \times 10^{4}\right.$, approximately for real examples in the last section).

In radial bearings, the Reynolds equation in turbulent regime has the same form that (2) but employing expressions (47) as viscosity coefficients. That is, in a stationary and isothermal case we have:

$$
\frac{\partial}{\partial \theta}\left(\bar{h}^{3} \bar{G}_{\theta} \frac{\partial \bar{p}}{\partial \theta}\right)+\frac{\partial}{\partial \bar{z}}\left(\bar{h}^{3} \bar{G}_{z} \frac{\partial \bar{p}}{\partial \bar{z}}\right)=\frac{\partial}{\partial \theta}\left(\frac{\bar{h}}{2}\right),
$$

with $\bar{G}_{z}=\bar{G}_{r}$ and $\bar{G}_{\theta}, \bar{G}_{r}$ given in (47).

\section{Industrial problem coupling axial and radial bearings}

In this section, a real application, that involves two bearings (axial and radial) working simultaneously in a rotating machine for ship propulsion (see Fig. 5), is presented. The device is designed to work totally submerged, using seawater as lubricant. The seawater low viscosity explains the small thermal effects involved in the fluid behavior, allowing isothermal studies of laminar and turbulent flows (even if the homogeneous temperature considered is relatively high). 


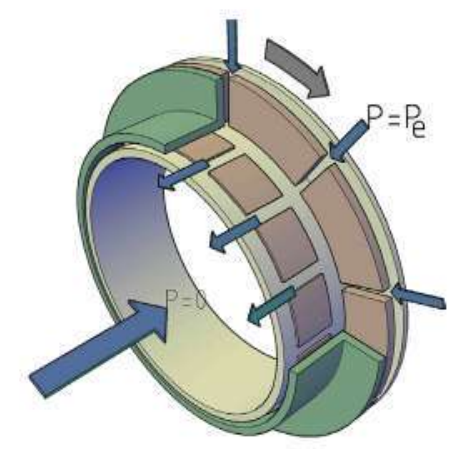

Fig. 5. Axial and radial bearings of the rotating machine.

Table 1

Thrust pad bearing and radial bearing data.

\begin{tabular}{llllll}
\hline Axial & Value & Radial & Value & Symbol & Value \\
\hline$R_{e}$ & $219 \mathrm{~mm}$ & $R_{a}$ & $170 \mathrm{~mm}$ & $\rho_{f}$ & $1025 \mathrm{~kg} / \mathrm{m}^{3}$ \\
$R_{i}$ & $166.5 \mathrm{~mm}$ & $L$ & $34 \mathrm{~mm}$ & $c_{f}$ & $4000 \mathrm{~J} /(\mathrm{kg} \mathrm{K})$ \\
$C$ & $0.1 \mathrm{~mm}$ & $C$ & $0.83 \mathrm{~mm}$ & $k_{f}$ & $0.6 \mathrm{~W} /(\mathrm{m} \mathrm{K})$ \\
$F_{\max }$ & $4.5 \mathrm{kN}$ & $F_{\max }$ & $250 \mathrm{~N}$ & $T_{0}$ & $273 \mathrm{~K}\left(0^{\circ} \mathrm{C}\right)$ \\
$\omega$ & $1315 \mathrm{rpm}$ & $\omega$ & $1315 \mathrm{rpm}$ & $\mu_{0}$ & $0.00188 \mathrm{Pas}$ \\
$T_{\text {ref }}$ & $35^{\circ} \mathrm{C}$ & $T_{\text {ref }}$ & $35^{\circ} \mathrm{C}$ & $\beta$ & $0.0236 \mathrm{~K}^{-1}$ \\
\hline
\end{tabular}

In this real problem a small reverse flow, from the outlet of the machine to the inlet through the small space between the rotating parts and the fixed parts, is observed. This fact implies the existence of a pressure on the external boundary of the thrust bearing that modifies the working conditions of the pads. When the device is operating the water flows from the gap to the radial bearing through the pad channels and the free space on the bend between thrust bearing and radial bearing (see Fig. 5). In this intermediate zone, an homogeneous pressure arises and needs to be computed. Finally, water seeps through the channels and through the gap between pads and the rotating impeller on the radial bearing.

Thus, the device simulation involves the solution of the hydrodynamic problem in both bearings with laminar or turbulent regime models, as presented in previous sections, and using the numerical methods there explained. Nevertheless, in this case we can assume that cavitation phenomena are absent. The reason is that this device is designed to operate in a bow tunnel, and then under a fairly large hydrostatic pressure. As a consequence, the relatively small depression originated in the bearing is not enough to originate cavitation.

The physical data values, used to compare results between laminar and turbulent models, and with or without inertial effects on the thrust bearing, are listed in Table 1 . In the case of the thrust bearing, the pads present a profile with a ramp being $C=0.1 \mathrm{~mm}$ from the inlet to the flat part. This flat part represents a $20 \%$ of width (see Fig. 3 ). The thrust. bearing consists of 10 pads with channels of $10 \mathrm{~mm}$ of width and $3 \mathrm{~mm}$ of depth. The radial bearing presents 8 supply grooves equally spaced on the upper half of the circumference (see Fig. 1), with $9.5 \mathrm{~mm}$ of width and $3 \mathrm{~mm}$ of depth. The rugosity coefficient of the materials in both bearings is 0.001 .

Fig. 6 shows the curves of load capacity versus minimum film thickness for the thrust bearing, considering laminar and turbulent flow regimes. Fig. 7 presents the corresponding load capacity curves versus eccentricity for the radial bearing (the horizontal line in both pictures denotes the required supported load at each bearing). Clearly the turbulent regime provides a higher load capacity in both cases. These numerical results were expected because load capacity is proportional to viscosity, and turbulence implies a higher effective viscosity. Inertial effects have not been considered here, since their influence turned out to be negligible. In fact, Fig. 8 shows the pressure on the pad with and without inertial effects in the turbulent flow regime, thus illustrating that the difference between pressures becomes unimportant.

For the solution of the problem involving both bearings working simultaneously, some previous considerations to perform the computing procedure are needed: pressure in the gap $P_{e}$ is not exactly known, but a reference value is given by the operation force in the machine, $F\left(4500 \mathrm{~N}\right.$ in this case). So, we take $P_{e}=\left(F /\left(\pi R_{c}^{2}\right)\right)$, where $R_{c}$ is the free radius of the cylindrical hole in the impeller (about $150 \mathrm{~mm}$ ). Intermediate pressure $P_{i}$ in the channel between axial 


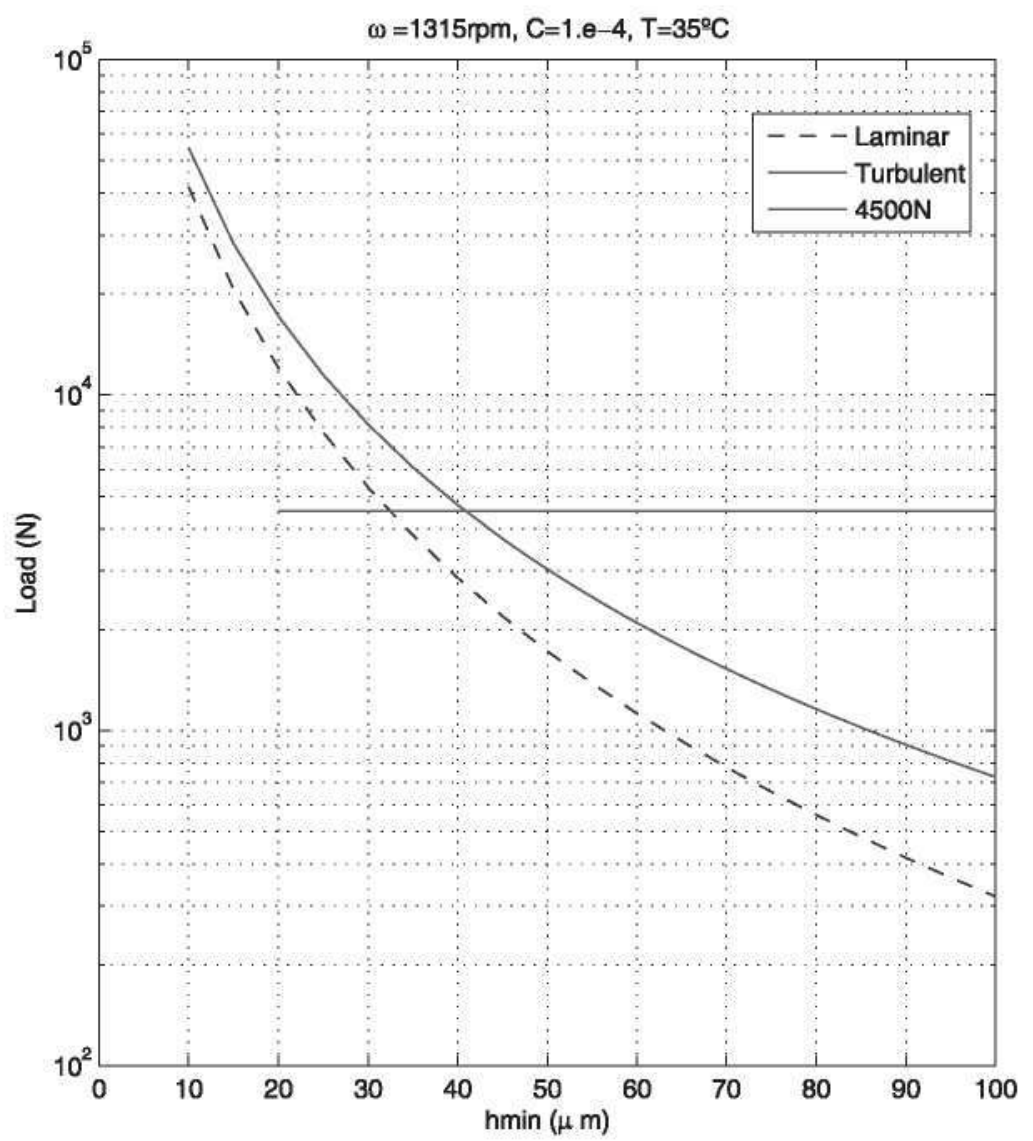

Fig. 6. Comparison of laminar and turbulent models: load capacity of the thrust bearing versus minimum film thickness (horizontal line is the reference load).

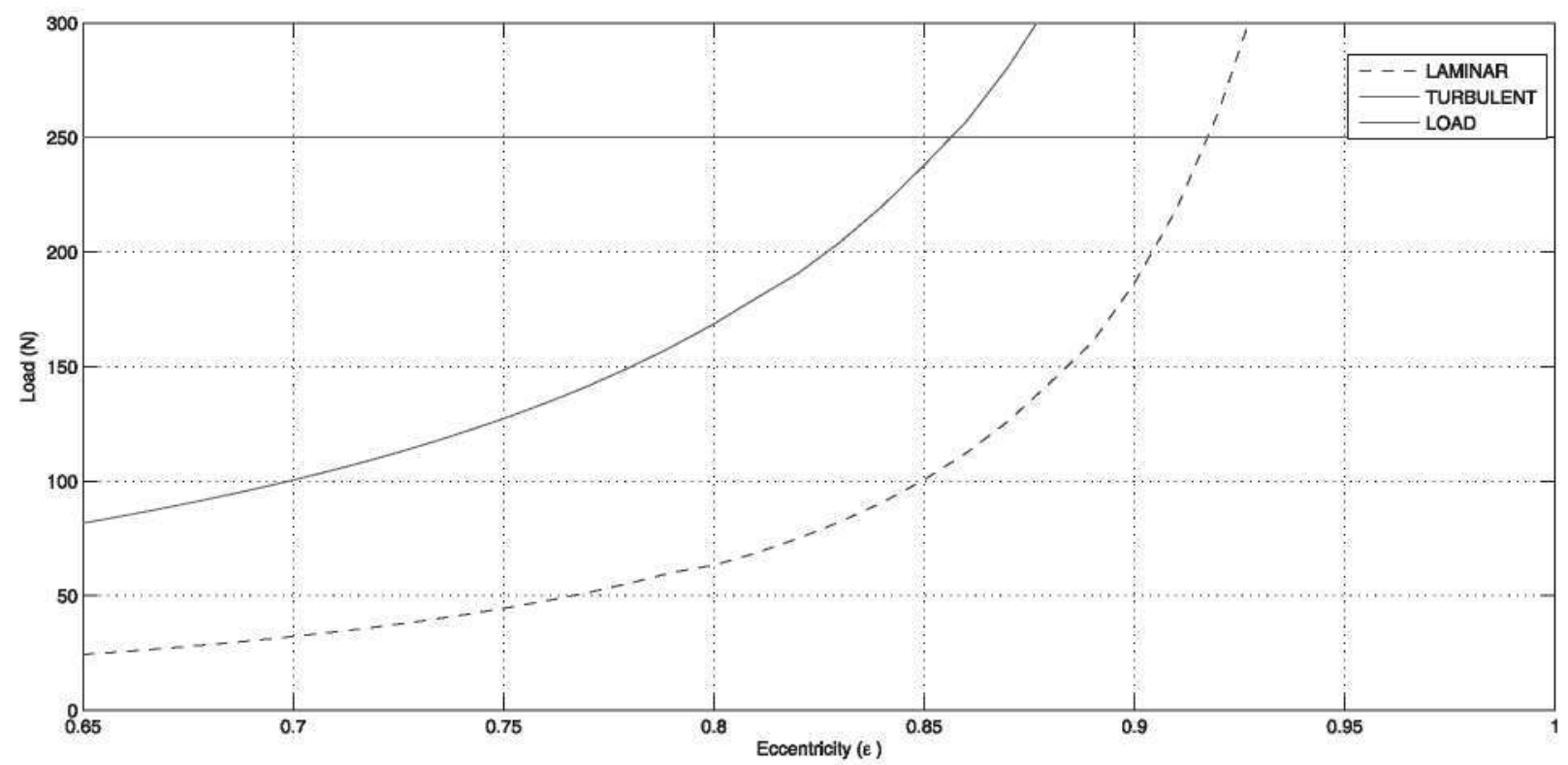

Fig. 7. Comparison of laminar and turbulent models: load capacity of the radial bearing versus eccentricity (horizontal line is the reference load). 


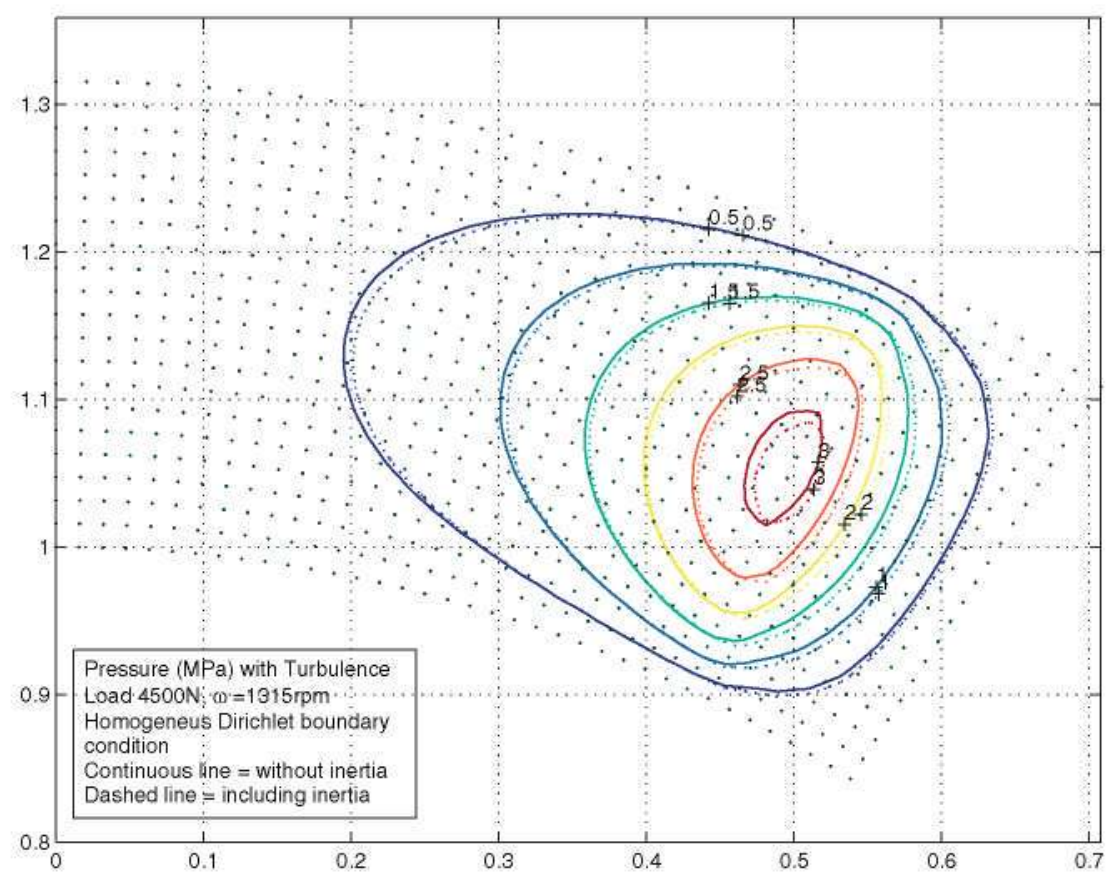

Fig. 8. Pressure in a pad with Dirichlet boundary conditions for turbulent flow with and without inertial effects.

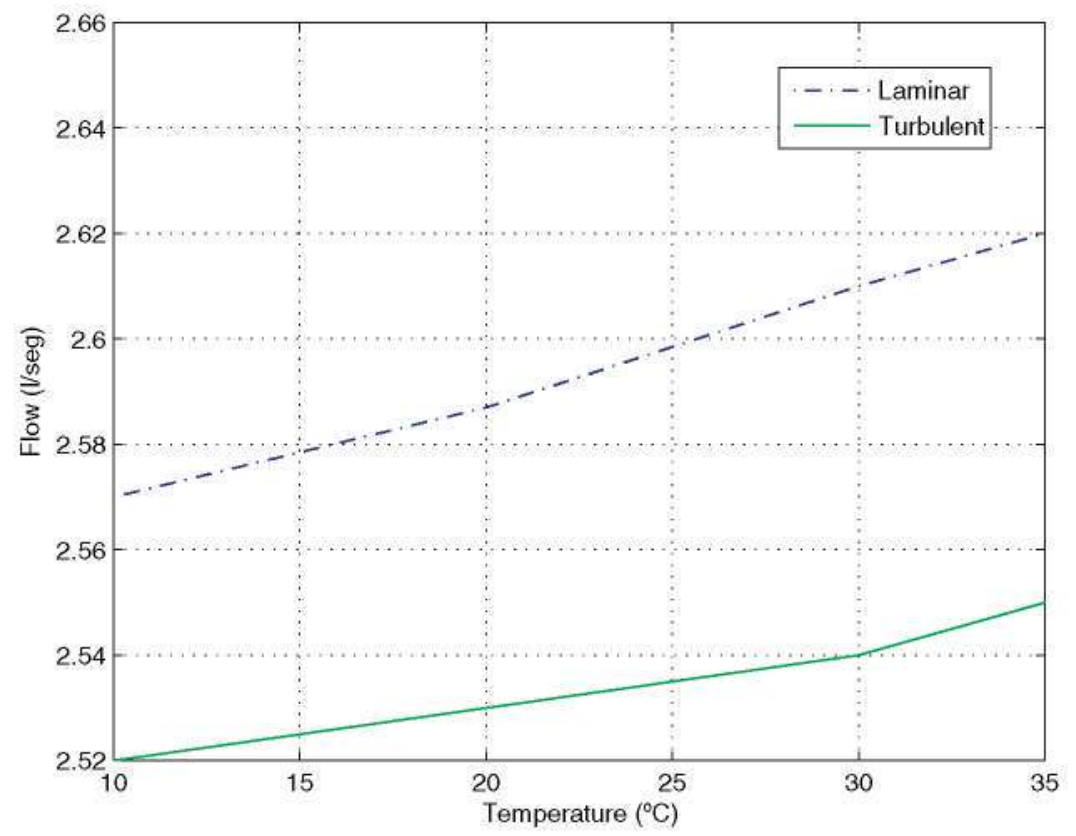

Fig. 9. Reverse flow calculated at different temperatures: laminar lubrication (dashed), turbulent lubrication (solid).

and radial bearings is another unknown. In order to obtain its value, an iterative algorithm is proposed. So, the pressure $P_{i}$ is updated at each iteration until the water flow through the thrust bearing equals the water flow through the radial bearing (see Fig. 5). Finally, the flow due to pressure differences in the channels (between pads on the thrust bearing and at supply grooves on the radial bearing) is computed by using an appropriate Moody's formula (see [15], among many other references) for closed channels. 


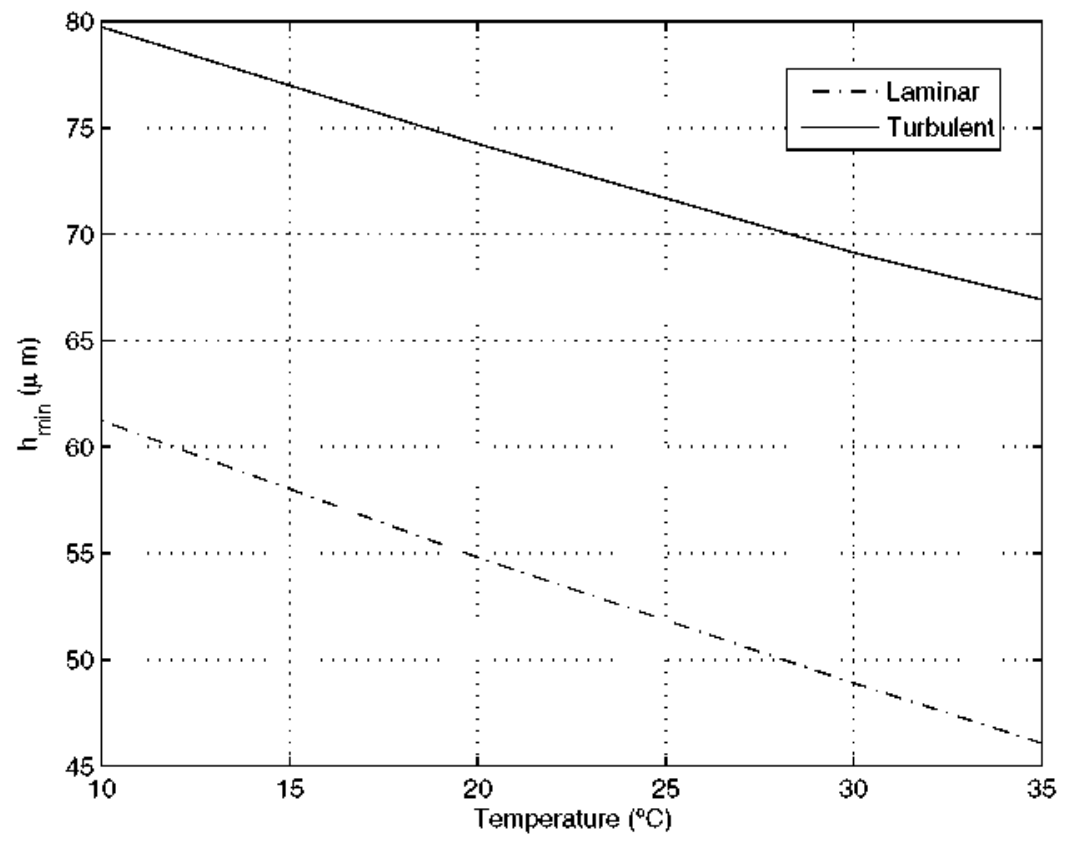

Fig. 10. Minimum film thickness in thrust pad bearing at different temperatures: laminar lubrication (dashed), turbulent lubrication (solid).

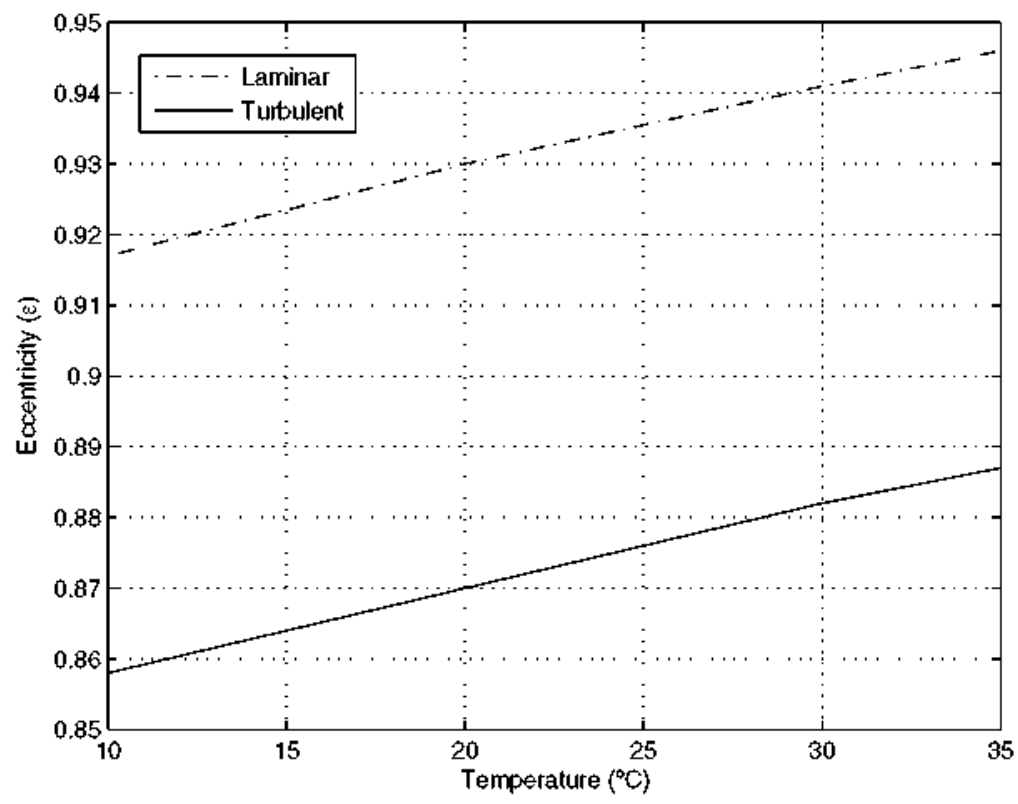

Fig. 11. Eccentricities of radial bearing at different temperatures: laminar lubrication (dashed), turbulent lubrication (solid).

With all this, the final Figs. 9-1 I show the flow, the minimum film thickness on the thrust bearing, and the eccentricity on the radial bearing at different temperatures. The numerical results provide clear differences between laminar and turbulent models when both bearings operate simultaneously.

\section{Conclusions}

The numerical simulations here presented show the good behavior of the global model for a lubrication problem in heavy regimes posed in this paper. The numerical method used to solve the coupled problem involves the computation 
of $2 \mathrm{D}$ temperature, pressure and cavitation, allowing to avoid many implicit constraints in the classical isothermal and stability analysis. Moreover, the comparison of hydrodynamic laminar and turbulent models provides a wide range of Reynolds numbers in the device operation conditions. So, these numerical methods are suitable for industrial applications with low viscosity lubricants. The proposed segregated algorithm results to be an efficient global numerical scheme due to its low computational cost and small storages.

Concerning the operation conditions of coupling axial and radial bearings in this real application, the following aspects can be concluded: the low viscosity of seawater leads to difficult operating conditions with both laminar and turbulent lubrication models (reduced minimum films thickness on the thrust pad bearing and high eccentricities on the radial bearing); thermal effects are small but not negligible; inertial effects are not important if total inertial or centrifugal inertia are considered.

In summary, the developed numerical code is a good tool for a quick design analysis (number of pads and their size, number of channels and their size, changes of the geometrical dimensions, etc.) under severe working conditions in rotating speed, temperature, and loads.

\section{References}

[1] G. Bayada, M. Chambat, Nonlinear variational formulation for a cavitation problem in lubrication, J. Math. Anal. Appl. 90 (1982) 286-298.

[2] G. Bayada, M. El Alaoui Talibi, C. Vázquez, Existence of solutions for elastohydrodynamic piezoviscous lubrication problems with a new model of cavitation, Eur. J. Appl. Math. 7 (1996) 63-73.

[3] G. Bayada, M. Chambat, C. Vázquez, Characteristics method for the formulation and computation of a free boundary cavitation problem, J. Comp. Appl. Math. 98 (1998) 191-212.

[4] G. Bayada, J. Durany, C. Vảzquez, Mathematical analysis of an elastohydrodynamic lubrication problem with cavitation, Math. Methods Appl. Sci. 18 (1995) 255-266.

[5] V.N. Constantinescu, S. Galetuse, Operating characteristics of journal bearings in turbulent inertial flow, J. Lubr. Technol. 104 (1982) $173-179$.

[6] J. Durany, G. García, C. Vázquez, Numerical simulation of a lubricated Hertzian contact problem under imposed load, Fin. Elem. Anal. Des. 38 (2002) 645-658.

[7] J. Durany, J. Pereira-Pérez, F. Varas, A cell-vertex finite volume method for thermohydrodynamic problems in lubrication theory, Comput. Methods Appl. Mech. Eng. 195 (44-47) (2006) 5949-5961.

[8] J. Durany, J. Pereira-Pérez, F. Varas, Numerical solution of steady and transient problems in thermohydrodynamic lubrication using a combination of finite element, finite volume and boundary element methods, Fin. Elem. Anal. Des. 44 (11) (2008) 686-695.

[9] J. Durany, J. Pereira-Pérez, F. Varas, Dynamical stability of journal-bearing devices through numerical simulation of thermohydrodynamic models, Tribol. Int. 43 (9) (2010) 1703-1718.

[10] H.G. Elrod, M.L. Adams, A computer program for cavitation, in: D. Dowson, M. Godet, C.M. Taylor (Eds.), Cavitation and Related Phenomena in Lubrication, Proceedings Mech. Engrg. Publ. Ltd., New York, 1975.

[11] J. Frene, D. Nicolas, B. Degueurce, D. Berthe, M. Godet, Lubrification Hydrodynamique, Eyrolles, Paris, 1990.

[12] P. Glendinning, Stability, Instability and Chaos: An Introduction to the Theory of Nonlinear Equations, Cambridge University Press, Cambridge, 1994.

[13] P.B. Kosasih, K. Tieu, An analysis of sector-shaped bearings operating in the transition regime, Wear 160 (1993) $291-299$.

[14] J.W. Lund, Review of the concept of dynamic coefficients for fluid film journal bearings, ASME Trans. J. Tribol. 109 (1) (1987) 37-41.

[15] D.B. Marghitu, Mechanical Engineer's Handbook, Academic Press, San Diego, CA, 2001.

[16] C.J. Myers, Bifurcation theory applied to oil whirl in plain cylindrical journal bearings, ASME J. Appl. Mech. 51 (1984) $244-250$.

[17] G. Quiao, L. Wang, T. Zheng, Linear stability analysis of a tilting-pad journal bearing system, ASME Trans. J. Tribol. 129 (2007) $348-353$.

[18] T.V. Rao, J.T. Sawicki, Stability analysis of a rough journal bearing considering cavitation effects, ASME Trans. J. Tribol. 127 (2005) $112-119$.

[19] J.K. Wang, M.M. Khonsari, Effects of oil inlet pressure and inlet position of axially grooved infinitely long journal bearings. Part I: analytical solutions and static performance, Tribol. Int. 41 (2008) 119-131.

[20] J.K. Wang, M.M. Khonsari, Effects of oil inlet pressure and inlet position of axially grooved infinitely long journal bearings. Part II: nonlinear instability analysis, Tribol. Int. 41 (2008) 132-140.

[21] Z. Xiao, L. Wang, T. Zheng, An efficient al gorithm for fluid force and its Jacobian matrix in journal bearing, ASME Trans. J. Tribol. 128 (2006) 291-295. 